\title{
POLYMERIC MATRICES FOR LASING DYES: RECENT DEVELOPMENTS
}

\author{
A. COSTELA $^{\text {a, }}{ }^{*}$, I. GARCIA-MORENO ${ }^{\text {a }}$, J. M. FIGUERA ${ }^{\text {a }}$, \\ F. AMAT-GUERRI ${ }^{\mathrm{b}}$ and R. SASTRE ${ }^{\mathrm{c}}$ \\ a Instituto de Química Física "Rocasolano", CSIC, Serrano 119, \\ 28006 Madrid, Spain; \\ ${ }^{\mathrm{b}}$ Instituto de Química Orgánica, CSIC, Juan de la Cierva 3, \\ 28006 Madrid, Spain; \\ ${ }^{\mathrm{c}}$ Instituto de Ciencia y Tecnología de Polimeros, CSIC, \\ Juan de la Cierva 3, 28006 Madrid, Spain
}

(Received 12 November 1997)

\begin{abstract}
An overview of recent results obtained by our group on the development of polymeric solid-state media incorporating either Rhodamine or Coumarin dyes is presented. The effect on the laser performance of the rigidity and viscoelastic properties of the polymeric matrix has been investigated. Lasing efficiencies similar to those found in ethanolic solution, and improved photochemical stability, as compared with that obtained in previous polymeric materials, were demonstrated for solid solutions of Rhodamine 6G (Rh6G) in appropriate copolymer formulations of 2-hydroxyethyl methacrylate (HEMA) and methyl methacrylate (MMA). Further improvements in photostability were obtained when modified Rh6G molecules were copolymerized with HEMA and MMA. When samples of this new highly homogeneous gain medium were placed in a rotating system, where the laser medium was scanned in a continuous way, a stable laser output with no sign of degradation after 500,000 shots was accomplished for transversal pumping at $337 \mathrm{~nm}$ with $1.7 \mathrm{~mJ}$ pulses from a $\mathrm{N}_{2}$-laser at $2 \mathrm{~Hz}$ repetition rate.

The effect on laser operation of different polymerization methods, dye concentration, and composition of the polymeric matrix was also investigated for Coumarin-doped gain media. Conditions were found that significantly improved both lasing efficiency and photostability in Coumarin 540A (C540A)-doped polymeric matrices. Insight into the main photodegradation mechanisms of this dye was provided by the study of fluorescence and lasing properties of a number of C540A solutions with increased viscosity, from liquid solution to solid polymer matrix.
\end{abstract}

Keywords: Dye; laser; solid-state dye laser; Rhodamines; Coumarins

*Corresponding author. e-mail: acostela@iqfr.csic.es 


\section{INTRODUCTION}

From the mid sixties, dye lasers have been attractive sources of coherent tunable visible radiation because of their unique operational flexibility. Features of dye lasers are broad tunability, with emission from the near ultraviolet to the near infrared; high gain; broad spectral bandwidth, which possibilitates the generation of ultrashort pulses; pulsed and continuous-wave operation, and wide variety of excitation sources [1]. Although dyes have been demonstrated to lase in the solid, liquid, or gas phases, liquid solutions of dyes in suitable organic solvents have been the most frequently used laser media becaused the active medium can be obtained in high optical quality and a liquid solution can be cooled by simply using a flow system. Nevertheless, the use of liquid solutions in dye lasers entrails a number of inconvenients, mainly related to the need of employing large volumes of organic solutions of dyes that are both toxic and expensive to use and dispose of. This, together with the need of complex and bulky cell designs for the continuous circulation of the solution has restricted the use of these laser systems outside the laboratory.

From the early days of development of dye lasers, attempts were made to overcome the problems posed by organic solvents by incorporating the dye molecules into solid matrices [2, 3]. A solidstate dye laser avoids the problems of toxicity and flammability, is compact, versatile, and easy to operate and maintain. Although this approach was very attractive, the first results were not very encouraging, with low lasing efficiencies and fast dye photodegradation, and as a result the research on solid-state dye lasers remained near dormant for over two decades. It was not until the late 1980's that developments of new and improved host materials with higher laserdamage resistance gave a new impulse to the field [4-6]. In recent years, the synthesis of new high-performance dyes and the implementation of new ways of incorporating the organic molecules into the solid matrix has resulted in significant advances towards the development of practical tunable solid-state dye lasers [7-20].

Although both inorganic glasses and polymeric materials have been successfully used as host matrices for lasing dyes, polymers offer a number of advantages both from the technical and economical points of view, which include high optical homogeneity [9], better chemical 
compatibility with organic dyes, control over medium polarity and viscosity in a way similar to conventional solvents [21], and adaptability to inexpensive fabrication techniques, which facilitate both miniaturization and the design of integrated optical systems. In addition, relevant properties of these materials, such as free volume, molecular weight and viscoelasticity, can also be modified in a controlled way [12, 22], providing desirable flexibility in the search for improved host materials for lasing dyes.

The two main problems found in polymeric solid-state dye lasers are usually the low laser-damage threshold of the material and the photodegradation of the dye. If these problems were properly addressed, the above mentioned advantages would make very attractive the use of polymers as host materials in solid-state dye lasers. In the early nineties our group began a research program in which the properties of dye-doped polymeric matrices were systematically studied, using the vast possibilities in polymer synthesis to modify the characteristics of the materials in a controlled manner, and designing new methods for the incorporation of the active dye molecules into the polymeric matrix. This approach evidenced to be very effective, and significant increases in both efficiency and photostability were accomplished [22, 23]. In this paper we present an up-to-date overview of some recent results obtained by our group on the development of polymeric solid-state dye lasers.

\section{MATERIALS}

\section{Dyes}

Most of our work in solid-state dye lasers has been performed with dyes of the Rhodamine and Coumarin families. The Rhodamine dyes, with emission in the yellow-red region of the spectrum, are known to give excellent laser results in liquid solution, so that they are an obvious first election in any attempt to develop a dye laser in the solid state. The well-known dye Rhodamine 6G (Rh6G, Scheme 1) was our most usual choice because there were numerous studies on this dye incorporated into different materials, which would facilitate the evaluation of our results by comparison with those previously reported 
<smiles>CCCNc1cc2oc3cc(=[NH+]CC)c(C)cc-3c(-c3ccccc3C(=O)OC)c2cc1OC</smiles>

Rhodamine 6G<smiles></smiles>

Rhodamine 640<smiles>CCCN1CCCc2c3c(C(C)(C)C)cc(c21)C(C(C)(C)C)=CC(=O)O3</smiles>

Coumarin 540A

SCHEME 1 Molecular structure of Rhodamine 6G, Rhodamine 640 and Coumarin 540A.

in the literature. Some experiments were performed using the more rigid Rhodamine 640 molecule (Rh640, Scheme 1) to analyze the effect of the structural rigidity of the dye on the laser operation.

The Coumarin dyes were chosen because of their emission in the technologically important blue-green spectral region. An efficient and photostable laser with emission in this region of the spectrum will find immediate applications in such important fields as optical storage or underwater communications. The dye Coumarin 540A (Scheme 1) was our first choice because of its high fluorescence quantum yield (0.98) when dissolved in poly (methyl methacrylate) [24].

\section{Polymers}

The basic requirements imposed on a polymeric host for lasing dye molecules are good optical transparency at both pump and lasing 
wavelengths, good solubility of the dye in the material, and resistance to pump laser radiation. The polymer poly(methyl methacrylate) (PMMA, Scheme 2) has been the most frequently used host for lasing dyes due to its excellent optical transparency in the visible and its relatively high laser-damage resistance. Nevertheless, some of the more usual dyes, such as Rh6G, have limited solubility in PMMA, and the structural rigidity of this polymer could be restraining its resistance to laser damage. Although there is no general consensus concerning the mechanism responsible for laser damage of polymeric materials, there is already enough evidence to establish that the laser damage of polymers is energy dependent, and that the matrix viscoelastic properties determine the damage resistance. Thus, it would be expected that it would be possible to significantly improve the laser resistance of the material by changing the viscoelastic properties of the medium in the appropriate way. Following this line of reasoning, it had been demonstrated that the external plasticization of the polymer, by adding low molecular weight dopants, improves significantly the laser resistance [25].

In our recent studies we tried a different but related approach, where the plasticity of the material was internally increased by copolymerization of methyl methacrylate (MMA) with 2-hydroxyethyl methacrylate (HEMA) (Scheme 2). The presence of HEMA as comonomer not only increases the plasticity of the material while maintaining the good transparency in the near-ultraviolet and visible spectral ranges, but also insures a good solubility of dyes such as Rh6G due to the polar character of HEMA.

Our experience with polymeric matrices incorporating laser dyes indicates that the lasing properties of a dye-polymer material depend largely on the methods used for their manufacture. These methods are

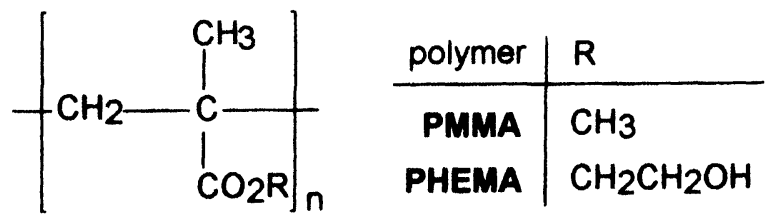

SCHEME 2 Molecular structures of PMMA and PHEMA. 
constrained by the stringent requirements placed upon the laser materials, which make the well-developed techniques for moulding polymeric materials not very well suited to ensure the high optical uniformity, absence of internal stress and purity of the final material needed in this application.

An alternative to the more conventional methods uses the uniform distribution of a liquid polymer plus dye solution in an organic solvent over the appropriate optical surface, followed by the subsequent removal by evaporation of the solvent. A first choice involves bulk radical polymerization of the initial monomer/fluorophore composition and subsequent machining and polishing of the material so obtained. This is the method we have used to prepare our materials.

Although each particular dye-monomer system requires the optimization of some specific polymerization conditions, it must be emphasized the need of a careful purification of both dye and monomer, the appropriate filtration or ultrafiltration of the resulting solution to be polymerized, and its $\mathrm{O}_{2}$-degassing in order to avoid the inhibition of the radical polymerization by atmospheric oxygen. Another important aspect of the polymerization process is the choice of an initiator and its concentration, that should affect neither the dye molecules nor the optical properties of the resulting material.

\section{EXPERIMENTAL SET-UP FOR LASER STUDIES}

The dye-doped lasing samples were cylindrical rods, $10 \mathrm{~mm}$ diameter $20 \mathrm{~mm}$ length, with a cut parallel to the axis of the cylinder defining a lateral flat surface of $\sim 4 \times 20 \mathrm{~mm}$. This surface as well as the ends of the rods were hand polished until reasonable flat surfaces were obtained. No attempt to produce laser grade surfaces was made. The dye concentration was chosen so that the optical density at the pump wavelength was about 10 , for $1 \mathrm{~cm}$ optical path. Absorption and fluorescence spectra of the different solid samples were obtained from thin strips (ca. $0.2 \mathrm{~mm}$ optical path) cut from the same samples used in the lasing experiments.

A schematic diagram of the laser system is shown in Figure 1. The oscillator cavity was formed by a $\approx 90 \%$ reflectivity flat aluminium mirror and the end face of the cylindrical sample as the output coupler, 


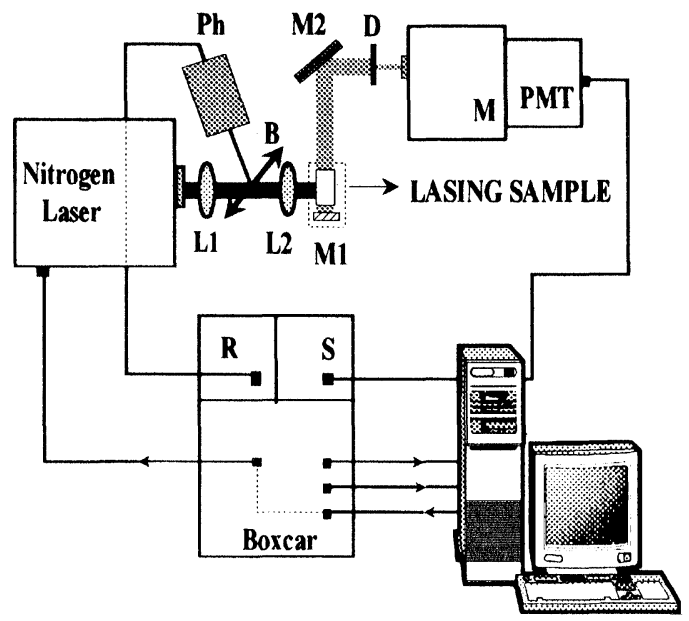

FIGURE 1 Schematic of the laser system experimental setup. B: beam splitter; D: Iris diaphragm; L1: spherical lens; L2: cylindrical lens; M: monochromator; M1 and M2: aluminium flat mirrors; Ph: photodiode; PMT: photomultiplier; R: reference; S: signal.

with typical cavity lengths of $4 \mathrm{~cm}$. We used a transversal pump scheme, with the exciting pulses being line-focused onto the lateral flat surface of the sample. Typical pump fluences were $30 \mathrm{~mJ} / \mathrm{cm}^{2}$. The airequilibrated polymer rods were usually pumped at $337 \mathrm{~nm}$ with millijoule pulses of nanosecond duration from a $\mathrm{N}_{2}$ laser. In some experiments the pump was a frequency-doubled Nd:YAG laser $(532 \mathrm{~nm})$. The highest pulse repetition rates used were $15 \mathrm{~Hz}$. Absolute pump and dye laser energies were measured with pyroelectric energy meters (not shown in Fig. 1). The estimated error in the energy measurements was $10 \%$.

\section{RHODAMINE-BASED GAIN MEDIA}

Rhodamine $6 \mathrm{G}$, probably the best known of all laser dyes, has been frequently investigated in solid-state dye lasers in a variety of solid hosts, on account of its high fluorescence quantum yield, low intersystem crossing rate, and low excited-state absorption at both pump and lasing wavelengths. Although previous studies on this molecule had provided many insights on the photodegradation 
mechanisms and photostability properties of the solid-state systems, by the beginning of the nineties the laser performance of Rh6G in solid matrices was still far away from that routinely obtained in liquid solutions. In particular, when incorporated into polymeric materials the laser emission lasted for no longer than a few hundred shots $[4,26]$.

\section{Rhodamine 6G Dissolved in Polymeric Media}

In a first study we were able to obtain significant, albeit insufficient, increases in both lasing efficiency and photostability by decreasing the polymer free volume ${ }^{* *}$ up to a certain point by controlled crosslinking of the polymeric chains with a diacrylic monomer [27]. These preliminary results demonstrated the important role played by the micro-structure of the host polymer on laser performance, and led us to further explore the effect of modifying the elasticity of the material.

Materials with increased flexibility were obtained by copolymerization of MMA with increasing proportions of HEMA [12]. A number of solid solutions of $\mathrm{Rh} 6 \mathrm{G}$ in the methacrylate copolymers were prepared, with HEMA:MMA vol/vol ratios of 1:9, 3:7, 1:1, 7:3 and 9:1. Appropriate amounts of Rh6G dye were dissolved in the different mixtures of freshly purified HEMA and MMA monomers. The resulting solutions were filtered into cylindrical polypropylene moulds that, after careful deaeration by bubbling dry argon, were sealed. Polymerizations were performed in the dark at $50^{\circ} \mathrm{C}$ for about one week.

When pumping at $337 \mathrm{~nm}$, broad-band laser emission with peak wavelength around $590 \mathrm{~nm}$ was obtained from all the samples studied [12], with efficiencies depending on the matrix composition, as shown in Figure 2. The highest lasing efficiency $(21.5 \%)$ was obtained with the HEMA:MMA 1:1 composition. This efficiency is similar to that obtained in liquid solutions of Rh6G in ethanol under equivalent experimental conditions.

The intensity of the laser output decreases with the number of pump pulses, with the lifetime (measured as the number of pulses that

\footnotetext{
** In a solid polymer the total volume per gram can be pictured as the sum of a "free volume" and an "occupied volume", this last including not only the volume of the molecules as represented by their van der Waals radii, but also the volume associated with vibrational motions.
} 


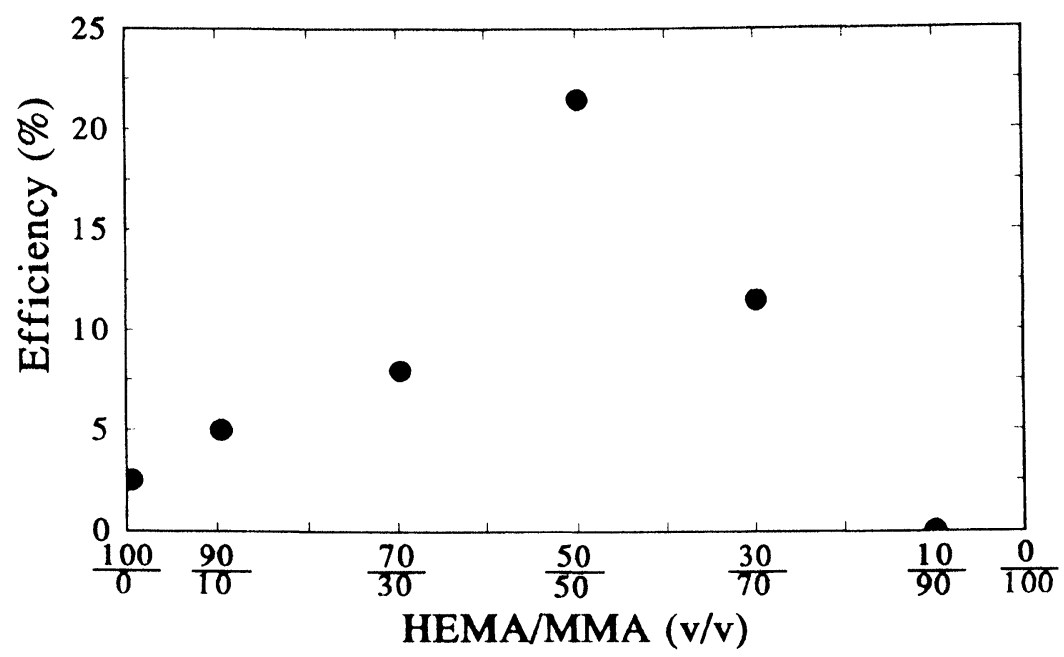

FIGURE 2 Energy conversion efficiency of Rh6G in various copolymers as a function of the HEMA:MMA ratio (vol/vol) in the polymeric matrix. $\mathrm{N}_{2}$-laser pump energy $(337 \mathrm{~nm}): 2.4 \mathrm{~mJ} /$ pulse. $[\mathrm{Rh} 6 \mathrm{G}]=1.0 \times 10^{-3} \mathrm{M}$.

produce a $80 \%$ drop in the laser output) depending on the composition of the polymeric matrix (Fig. 3) and correlating with the lasing efficiency. Thus, the highest photostability was obtained with the copolymer P(HEMA:MMA 1:1), with a lifetime of 8500 pulses for pumping at repetition rates of up to $15 \mathrm{~Hz}$ (Fig. 3).

When the Rh6G/P(HEMA:MMA 1:1) samples were pumped at $532 \mathrm{~nm}$, the peak of the laser emission shifted to $568 \mathrm{~nm}$ and the lifetime increased to 45000 pulses [12] (Fig. 4). This improvement in photostability could be related to the lower energy difference between the pump and laser photons in the $532 \mathrm{~nm}$ experiment, so that the energy released to the medium as heat is minimized, with resulting lower thermal degradation. Thus, these results can demonstrate large local rises in temperature as the main cause of degradation of the dye molecules, as had been previously suggested [28]. In addition, the more energetic ultraviolet $337 \mathrm{~nm}$ light may photodegradate not only dye molecules but also polymer chains.

The behaviour of the copolymers, summarized in Figure 2, could be understood in terms of an internal plasticization mechanism. It has been shown [29-31] that for good damage resistance the induced 


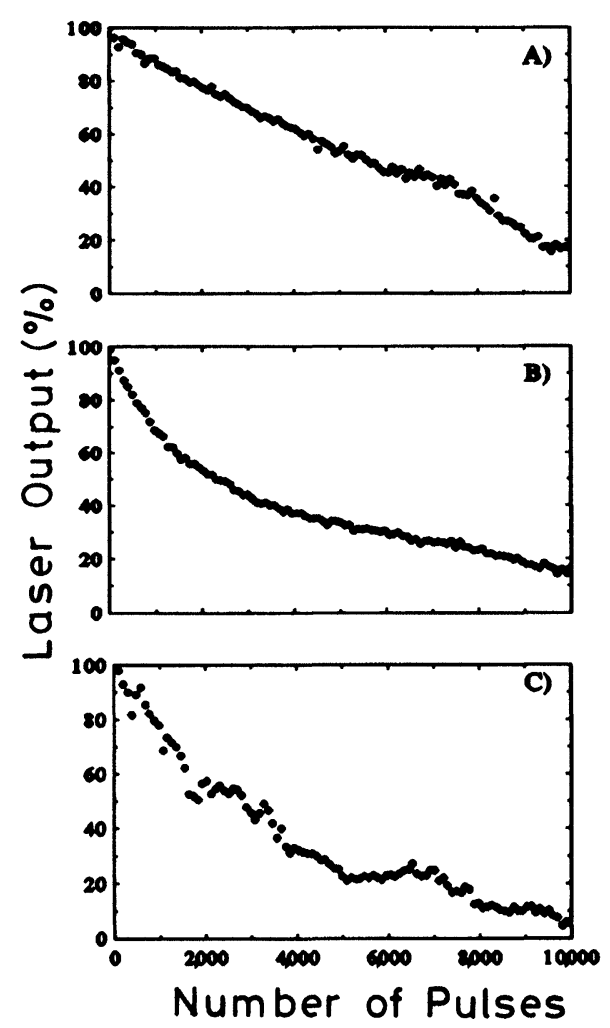

FIGURE 3 Normalized laser output as a function of the number of pump pulses at different repetition rates for $1.0 \times 10^{-3} \mathrm{M}$ Rh6G in various copolymers: (A) Rh6G/ P(HEMA:MMA 1:1), $2 \mathrm{~Hz}$; (B) Rh6G/P(HEMA:MMA 1:1), $15 \mathrm{~Hz}$; (C) Rh6G/ P(HEMA:MMA 3:7), $2 \mathrm{~Hz} . \mathrm{N}_{2}$-laser pump energy $(337 \mathrm{~nm}): 1.2 \mathrm{~mJ} /$ pulse. For clarity, only one of every 100 pulses has been plotted.

elastic limit of the material must be smaller than its brittle-fracture limit. Plasticization lowers the elastic limit, and it has been observed [31] that modifying the polymer host by copolymerization of MMA with appropriate aliphatic acrylic monomers (internal plasticization) can reduce the induced elastic limit to below the brittle-fracture point, improving the material's resistance by orders of magnitude. In our case, the polymeric material becomes more plastic, and hence more photostable, as the HEMA comonomer concentration increases. On the other hand, as the internal plasticization increases, the "polymer 


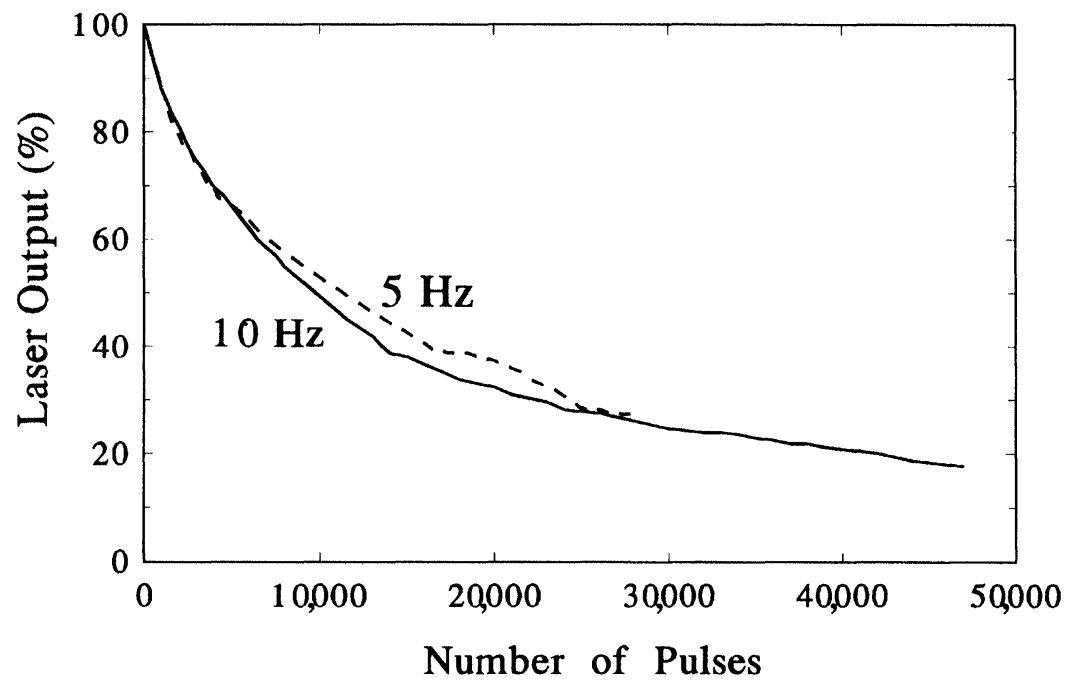

FIGURE 4 Normalized laser output as a function of the number of pump pulses at different repetition rates for $8.35 \times 10^{-5} \mathrm{M}$ Rh6G in P(HEMA:MMA 1:1) pumped at $532 \mathrm{~nm}$. Pump energy: $1.7 \mathrm{~mJ} /$ pulse.

cage" that surrounds the dye groups is less protective (the polymer becomes less rigid) and, as result, the chromophore is more easily bleached [32]. Thus, there should be a MMA: HEMA composition that represents the best compromise between these two conflicting requirements.

\section{Rhodamine 640 Dissolved in HEMA:MMA Copolymer}

Having found a material with a good balance between rigidity and flexibility, next we proceeded to study the effect on lasing properties of the structural rigidity of the dye molecule. To this end, we analysed the laser behaviour of Rhodamine 640 [33] (Rh640), a dye related to $\mathrm{Rh} 6 \mathrm{G}$ but with a more rigid structure (Scheme 1). We reasoned that the higher rigidity of the molecular structure of Rh640 could inhibit non-radiative decay processes, reducing the amount of energy that appears locally as heat and, thus, improving the photostability with respect to that of $\mathrm{Rh} 6 \mathrm{G}$.

Contrary to our expectations, when samples of Rh640 dissolved in P(HEMA:MMA 1:1) were tested, lasing efficiencies of only $4 \%$ and 
lifetimes of at most 800 pulses ( $337 \mathrm{~nm}$ pumping) were obtained. This important decrease in efficiency and photostability with respect to that of $\mathrm{Rh} 6 \mathrm{G}$ in the same copolymer is likely indicating that the microstructure of the host polymer, being optimized for Rh6G, is not adequate for Rh640. The molecular volume of Rh640 is larger than that of Rh6G, and to optimize the polymer-cage protecting effect it could be required a polymer with a larger free volume. In addition, the higher rigidity of $\mathrm{Rh} 640$ could be compensated with an increased internal plasticization of the polymeric material. These results indicate that for each dye there is an optimum copolymer composition that results in the best matrix/dye combination.

\section{Modified Rhodamine 6G Copolymerized with Methacrylic Monomers}

As the main cause of degradation of the dyes incorporated into polymeric matrices seemed to be the thermal destruction of the dye due to poor thermal dissipation in the polymer host, molecular modifications facilitating the dissipation of the absorbed energy which is not converted into light emission should avoid early degradation. One such modification can be the covalent linkage of the dye to the polymeric chain, that would provide additional channels for the energy elimination along the polymer backbone, with the corresponding increase in the laser's photostability. In addition to the effect of improving the dissipation of energy, the covalent bonding of a chromophore to a polymer chain must restrict its mobility, decreasing the rate of biomolecular reactions leading to photochemical degradation, hindering the internal micromotions of the excited fluorophores, and avoiding non-emissive deactivations via rapid internal rotations or conformational changes.

To apply the above ideas to rhodamines, it was first necessary to modify the $\mathrm{Rh} 6 \mathrm{G}$ molecules so that polymerizable double bonds were incorporated in the appropriate positions. Two new monomers containing the Rh6G chromophore and an allyl or a methacryloyl group in the same molecule were synthesized ( $\mathrm{Rh}-\mathrm{Al}$ and $\mathrm{RhBzMA}$, respectively; Scheme 3) and, subsequently, copolymerized with mixtures of HEMA and MMA [34]. The preparation of the 
<smiles>[R2]C(=O)c1ccccc1-c1c2cc(C)c(=[NH+]CC)cc-2oc2cc(NCCC)c(C)cc12</smiles>

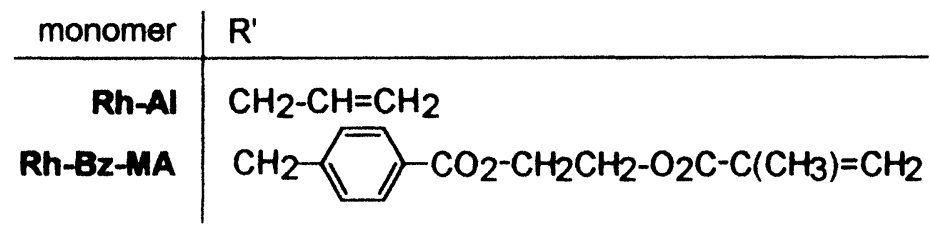

SCHEME 3 Molecular structure of compounds Rh-Al and Rh-Bz-MA.

polymerized dye laser samples involves mixing a suitable amount of the polymerizable dye monomer with freshly purified HEMA, placing the mixture in an ultrasonic bath until complete dissolution of the dye, and then adding appropriate amounts of pure MMA and free radical initiator, 2,2'-azobis(isobutyronitrile) (AIBN). After filtering and deaeration, polymerizations were performed in the dark at $40^{\circ} \mathrm{C}$ for two days, and then at $45^{\circ} \mathrm{C}$ for about one day. The temperature was then raised to $60^{\circ} \mathrm{C}$ and increased slowly up to $80^{\circ} \mathrm{C}$ over a period of several days, in order to decompose residual AIBN. Finally, the temperature was reduced in steps of $5^{\circ} \mathrm{C}$ per day until room temperature was reached.

A summary of the data obtained with some of the new terpolymers [34] is shown in Table I, where the previous results obtained with Rh6G dissolved in the copolymer P(HEMA:MMA 1:1) are also included for comparison. In Figure 5 the effect of the repetition rate on the laser lifetime is shown graphically for two of the materials.

The lasing properties of the terpolymer with the allyl ester of Rh6G as comonomer are clearly worse than those of the corresponding model dye dissolved in P(HEMA:MMA 1:1) (Tab. I), whereas the terpolymers containing the methacryloyl-substituted Rh6G as comonomer maintain the lasing efficiency and improve the photostability 
TABLE I Laser parameters * for dyes of the Rhodamine $6 \mathrm{G}$ family either dissolved in copolymers of HEMA and MMA or covalently bound to similar copolymers. Chromophore concentration: $10^{-3} \mathrm{M}$. $\mathrm{N}_{2}$-laser pump energy: $1.2 \mathrm{~mJ} /$ pulse

\begin{tabular}{|c|c|c|c|c|c|c|c|}
\hline Material & $\begin{array}{l}\lambda_{\max } \\
(\mathrm{nm})\end{array}$ & $\begin{array}{c}\Delta \lambda \\
(n m)\end{array}$ & $\begin{array}{c}E_{p, t} \\
(m J)\end{array}$ & $\begin{array}{l}\text { Eff } \\
(\%)\end{array}$ & $\begin{array}{r}\text { Lifeti } \\
15 \mathrm{~Hz} 10 \mathrm{~Hz}\end{array}$ & $\begin{array}{l}m e^{\dagger} \\
5 \mathrm{~Hz}\end{array}$ & $2 \mathrm{~Hz}$ \\
\hline Rh6G/P(HEMA:MMA 1:1) & 593 & 13 & 0.48 & 21 & 8500 & 8500 & 8500 \\
\hline P[RhAl(HEMA:MMA 1:1)] & 589 & 12 & 0.48 & 11 & 4500 & 6000 & 6000 \\
\hline P[RhBzMA(HEMA:MMA $1: 1)]$ & 587 & 11 & 0.58 & 18 & 900010500 & 10500 & 13500 \\
\hline P[RhBzMA(HEMA:MMA 7:3)] & 593 & 10 & 0.65 & 18 & 8000 & 9000 & 20000 \\
\hline
\end{tabular}

${ }^{*} \lambda_{\max }$ : peak of the laser emission; $\Delta \lambda$ : FWHM of the laser emission; $\mathrm{E}_{p, t}:$ minimum pump energy producing laser emission (pump threshold); Eff: energy conversion efficiency.

${ }^{\dagger}$ Number of pulses that produces an $80 \%$ drop in the laser output.

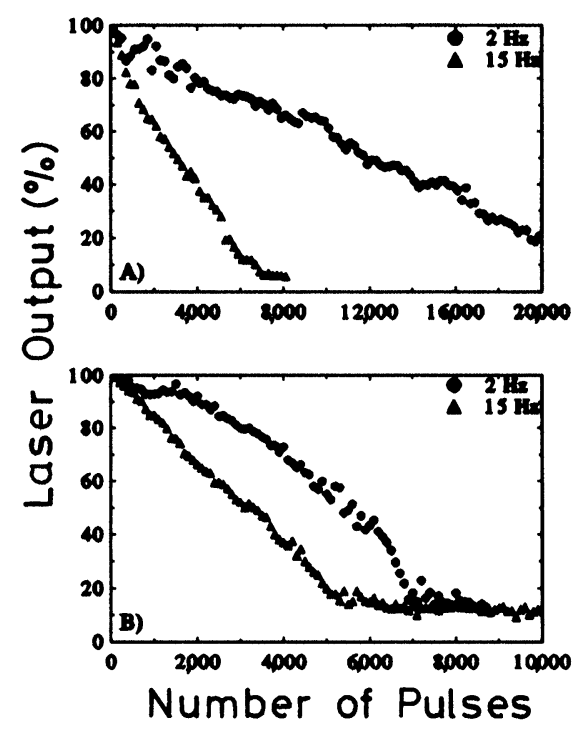

FIGURE 5 Normalized laser output as a function of the number of pump pulses at different repetition rates for P[RhBzMA (HEMA:MMA 7:3)] (A), and P[RhAl(HEMA:MMA 1:1)] (B). $\mathrm{N}_{2}$-laser pump energy: $1.2 \mathrm{~mJ} /$ pulse. For clarity, only one of every 100 pulses has been plotted. Notice the different $\mathrm{X}$ axis scales.

with respect to the solid solution, with lifetimes of up to 20,000 shots at $2 \mathrm{~Hz}$ repetition rate. Thus, the covalent linkage of the dye to the polymeric chain is producing the expected results only in the case of the terpolymers with the RhBzMA comonomer. Although no single cause is expected to fully explain the behaviour of these rather 
complicated polymer systems, it seems, in a first approximation, that the distance between the chromophore's functional group and the main polymeric chain (Scheme 3) should play a role in the different behaviour between the two comonomers. In the terpolymers with the $\mathrm{RhAl}$ comonomer, the chromophore's functional group is close to the polymeric main chain. This could result in interactions between the excited dye chromophores and the macromolecules, with electronic energy transfer to the polymer main chain and the formation of free radicals [22]. These active radicals could in turn interact with the dye groups and stimulate their destruction. In the terpolymers with the methacryloyl-substituted Rh6G as comonomer, the chromophore's pendant group is far from the main polymeric chains, resulting in no direct interaction. In this case, the predominant effect is the improved dissipation of the excess absorbed energy, through the additional channels provided by the covalent linkage of the dye to the polymeric chain, and the resulting improvement in photostability. At low repetition rates $(2 \mathrm{~Hz})$, this increase in photostability is particularly noticeable, whereas at $15 \mathrm{~Hz}$ repetition rate there is no difference in lifetime between the two terpolymers P[RhBzMA(HEMA:MMA)] and the solid solution of Rh6G in the 1:1 copolymer of HEMA and MMA. It seems that at high repetition rates the extra dissipative channels in the terpolymer are not able to dissipate the excess energy faster enough. Thus, the material loses its main advantage over the solid solution of Rh6G, and the stability is comparable in both cases.

In order to demonstrate the potential of these materials as active media in commercial solid-state dye lasers, experiments were performed where P[RhBzMA(HEMA:MMA 7:3)] laser rods of $20 \mathrm{~mm}$ diameter and $20 \mathrm{~mm}$ length were placed in a rotating system where the sample is scanned in a continuous way [13]. When pumping transversely at $337 \mathrm{~nm}$ with $1.2 \mathrm{~mJ}$ pulses from a $\mathrm{N}_{2}$-laser at $2 \mathrm{~Hz}$ repetition rate, it was obtained stable laser emission with no sign of degradation in the laser output after 500,000 shots [16] (Fig. 6).

Important increases in both efficiency and photostability of polymeric solid-state dye lasers have been reported [7, 11]. Using "modified acrylic plastic" doped with pyrromethene. $\mathrm{BF}_{2}$ dyes pumped at $532 \mathrm{~nm}$ by a frequency-doubled Nd:YAG laser in a longitudinal configuration, Hermes et al. [7] reported slope efficiencies of $85 \%$ and useful lifetimes (when the initial output energy of the laser 


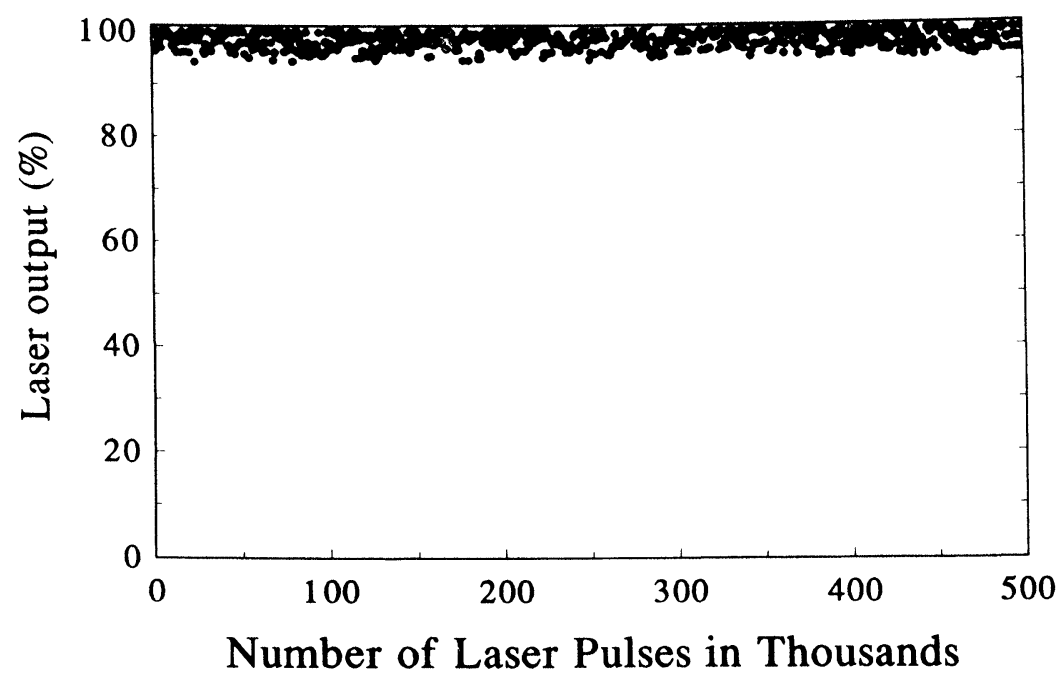

FIGURE 6 Normalized laser output as a function of the number of laser pulses from P[RhBzMA(HEMA:MMA 7:3)] samples placed in a rotating system. Pumping was at $337 \mathrm{~nm}$, with $1.2 \mathrm{~mJ}$ pulses from a $\mathrm{N}_{2}$-laser at $2 \mathrm{~Hz}$ repetition rate. For clarity, only one of every 800 pulses has been plotted.

was reduced by about one third) greater than 20,000 shots at $3.33 \mathrm{~Hz}$. Maslyukov et al. [11] used Rhodamine-based dyes in modified poly (methyl methacrylate) (MPMMA) obtaining efficiencies in the range $40-60 \%$, depending on the dye concentration. Once more, the pump configuration was longitudinal, with a Q-switched frequency-doubled Nd:YAG laser providing 10 -ns pump pulses at $532 \mathrm{~nm}$. These authors also found that both the conversion efficiency and the useful lifetime decreased dramatically with increasing pump-repetition rate: at $20-\mathrm{Hz}$ repetition rate the conversion efficiency was 4-5 times lower than that at $3.33-\mathrm{Hz}$, and the useful lifetime (measured as a $50 \%$ efficiency drop) was only approximately 600 shots.

Our results summarized in Table I show that the photostability of the Rh6G chromophore incorporated into adequate polymeric materials is higher than that of the Rh-based dyes incorporated into MPMMA, and compares well with that described for pyromethene. $\mathrm{BF}_{2}$ dyes, such compounds being intrinsically more photostable than Rh6G [7]. In this regard, it is necessary to take into account that we are pumping the samples at $337 \mathrm{~nm}$ in a transversal configuration. As 
shown previously (Fig. 4) a substantial lifetime increase is to be expected if the pump wavelength is changed from $337 \mathrm{~nm}$ to $532 \mathrm{~nm}$.

\section{Related Studies}

For the last years we have been collaborating with other groups to further explore the possibilities of the materials we are developing. In particular, the group of Prof. Guerra (Universidad Complutense, Madrid, Spain) investigated flashlamp-pumping of solid-state dyedoped materials [35], and F. J. Duarte (Eastman Kodak Co., Rochester, NY, USA), in collaboration with J. J. Ehrlich and T. S. Taylor (US Army Missile Command, Redstone Arsenal, AL, USA), built and tested dispersive laser oscillators to be used with the new materials [20].

When a laser rod (14 mm diam. and $267 \mathrm{~mm}$ length) of $\mathrm{Rh} 6 \mathrm{G} /$ P(HEMA:MMA 1:1), placed in a simple resonator made up of two flat mirrors (total reflector and 30\% reflector, respectively), was pumped with a xenon coaxial flash-lamp with UV and blue light filtered out [35], laser emission with pulses of up to $500 \mathrm{~ns}$ duration (FWHM) were obtained. The average excitation energy was $150 \mathrm{~J}$ per pulse, and during the measurements the rod was irradiated by more than 30 pumping shots, with no noticeable degradation of the output observed. Thus, the solid-state sample has remarkable photostability, as compared to other reported solid-state dye materials [24, 36, 37].

When samples of Rh6G/P(HEMA:MMA 1:1) and P[RhBzMA(HEMA:MMA 7:3)] in a wedge-type geometry $(20 \mathrm{~mm}$ diam. and trapezoidal cross-section) were placed in a narrow-linewidth multiple-prism grating oscillator [20], laser emission with $4-5 \%$ efficiency and linewidth $\leq 1.8 \mathrm{GHz}$ for double-longitudinal-mode emission was obtained. The pumping was semilongitudinal at $532 \mathrm{~nm}$ and $1 \mathrm{~Hz}$ repetition rate, with a cavity length of $80-85 \mathrm{~mm}$. Measured beam divergence was in the $2-2.3 \mathrm{mrad}$ range. Although thermal lensing leads to an increment in beam divergence, as compared to that obtained in a liquid gain medium, it induces oscillation in the unstable resonator regime, thus contributing to the attainment of $\mathrm{TEM}_{00}$ beam profiles. Threshold energy densities for dye bleaching were measured to be about $0.7 \mathrm{Jcm}^{-2}$, which provides a comfortable margin for the design and operation of narrow-linewidth oscillators. 


\section{COUMARIN-DOPED GAIN MEDIA}

Coumarins are a class of laser dyes with emission in the blue-green region of the spectrum. Although some of these dyes have demonstrated to lase efficiently in liquid solution, no much work has been done on solid-state dye lasers based on Coumarins or other molecules with emission in the blue. After some early studies using a variety of molecules in a number of host media, with not very promising results [38-43], some improvements in laser performance were reported by Itoh et al. [44] and Pacheco et al. [24] with coumarin-doped PMMA media. Nevertheless, it was not until the beginning of the nineties that some detailed studies appeared on the lasing properties of different dyes emitting in the blue and green $[6,17,45-51]$ although in all cases the dyes were incorporated into sol-gel materials. By the same time, we began to investigate the lasing properties in polymeric matrices of a new family of laser dyes, with emission at wavelengths in the bluegreen region, in which the generation of stimulated radiation is due to an intramolecular proton-transfer photochemical mechanism [52]. When the new dyes were copolymerized with methacrylic monomers and pumped transversely with pulses from a $\mathrm{N}_{2}$ laser, energy conversion efficiencies of $11 \%$, similar to those obtained in liquid solution, were obtained, but the lifetime was only about one hundred shots [28]. Thus, in order to improve the laser photostability in this important spectral region, we began a systematic investigation on the lasing properties of polymeric materials incorporating dyes of the Coumarin family.

The dye Coumarin 540A (C540A, Scheme 1) was incorporated into two-different polymeric matrices [53]; a 1:1 v/v copolymer of HEMA and MMA, and a pure PMMA homopolymer. AIBN was used as thermal free radical initiator, and polymerizations were performed in the dark at $40^{\circ} \mathrm{C}$ for about 62 hours, and then at $50^{\circ} \mathrm{C}$ for two days. Under these conditions lasing efficiencies of $8 \%$ and $9.5 \%$ were obtained for C540A/P(HEMA:MMA 1:1) and 540A/PMMA, respectively, with lifetimes of less than 500 shots (Fig. 7, curves B and C), for a dye concentration of $3 \times 10^{-2} \mathrm{M}$ and transversal pumping at $337 \mathrm{~nm}$ with pulses of $1.2 \mathrm{~mJ}$ at $2 \mathrm{~Hz}$ repetition rate. When the solid solution of C540A in PMMA was prepared by a method involving reduced concentration of initiator and gentle thermal treatment $\left(35^{\circ} \mathrm{C}\right.$ during 


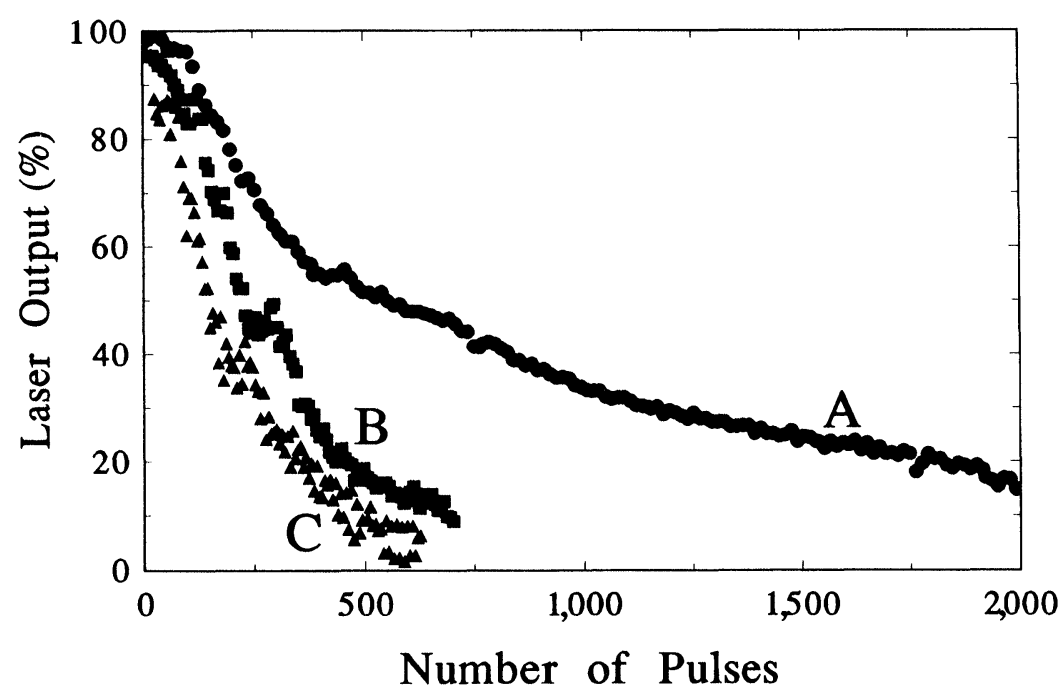

FIGURE 7 Normalized laser output as a function of the number of pump pulses for solid solutions of C540A in: (A) slowly polymerized PMMA; (B) normally polymerized PMMA; (C) P(HEMA:MMA 1:1). $\mathrm{N}_{2}$-laser pump energy and repetition rate: $1.2 \mathrm{~mJ} /$ pulse and $2 \mathrm{~Hz}$, respectively. Dye concentration: $3 \times 10^{-2} \mathrm{M}$.

two days, followed by two days at $40^{\circ} \mathrm{C}$ and a week at $45^{\circ} \mathrm{C}$ ), that results in the polymerization taking place at very slow rate, an efficiency of $11 \%$ and a lifetime of 2000 pulses (Fig. 7, curve A) were obtained. These results improve the laser efficiency by a factor of $\approx 2$ and the photostability by two orders of magnitude with respect to the best performance based on Coumarin-doped PMMA materials reported to date [24].

Further improvements in photostability would require a greater understanding of the degradation mechanisms involved when these fluorophores are embedded in polymeric matrices. To this end, we are proceeding in two directions. On the one hand, in a very recent work [21] we have investigated the fluorescence and lasing properties of a number of $\mathrm{C} 540 \mathrm{~A}$ solutions with increased viscosity, from liquid solution in 1,4-dioxane to solid solution in PMMA, with solutions of intermediate viscosity obtained by dissolving appropriate amounts of PMMA in 1,4-dioxane. It was found that the fluorescence quantum yield and lasing efficiencies are highly dependent on the free-volume of 
the medium, decreasing as the viscosity of the solution increases. This behaviour could be reflecting the strong influence of the rigidity of the medium on the radiative processes, whilst the non-radiative decay seems to be almost independent of the solution viscosity, probably due to the rigid structure of the Coumarin molecule that inhibits the torsional decay route.

The photodegradation mechanisms acting on the fluorophores were analyzed by following the dependence of laser induced fluorescence and laser output on the number of pump laser pulses. The laser photostability appears to be a function of the elasticity of the medium surrounding the dye molecules. The main photodegradation mechanisms of the dye were identified to be photothermal and photobleaching processes, that result in the irreversible and growing presence of decomposition products which absorb at the laser emission wavelength. The rate of recovery of the fluorescence intensity after irradiation was also investigated in order to obtain information on the mobility of the dye molecules in the different samples. By using Fick's second law, diffusion coefficients of the dye molecules as a function of the increased viscosity of the medium were determined.

In the above studies on C540A-doped polymeric matrices, it was found that, contrary to the behaviour of Rh6G, dissolving C540A in a HEMA:MMA copolymer results in a decrease of both laser efficiency and photostability, as compared with the C540A/PMMA material, in a new demonstration that for each dye there is an optimum copolymer composition. In the present case this behaviour could be partly due to the change in the polarity of the medium induced by the presence of HEMA, as it has been experimentally demonstrated in liquid solutions [54], where an increase in the polarity of the Coumarin solvent leads to a clear decrease in laser emission efficiency. Nevertheless, a thorough study of the influence of the medium's plasticity on laser operation in Coumarin-doped gain media would be an important second line of attack in our effort to improve laser performance in these media. Thus, work is in progress where the effect on laser operation of relaxing the structural rigidity of both dye molecule and polymeric media is studied. To this end, the dye Coumarin 503 (C503), where the amine substituent has a free rotor character providing the molecule with structural mobility, is used, and the influence on laser operation of dye concentration, viscoelasticity of the matrix, and degree of crosslinking 
of the polymeric chains is to be examined, as well as the effectiveness of the addition of chemical stabilizers of the dye molecules.

\section{Acknowledgements}

The work described in the present review was supported by Project No. MAT94-0757 of the Spanish CICYT.

\section{References}

[1] Duarte, F. J. and Hillman, L. W. (Eds), Dye Laser Principles (Academic Press, New York, 1990).

[2] Soffer, B. H. and McFarland, B. B. (1967). Appl. Phys. Lett., 10, 266.

[3] Peterson, O. G. and Snavely, B. B. (1968). Appl. Phys. Lett., 12, 238.

[4] Gromov, D. A., Dyumaev, K. M., Manenkov, A. A., Maslyukov, A. P., Matyushin, G. A., Nechitailo, V. S. and Prokhorov, A. M. (1985). J. Opt. Soc. Am. B, 2, 1028.

[5] Salin, F., Le Saux, G., Georges, P., Brum, A., Bagnall, C. and Zarzycki, J. (1989). Opt. Lett., 14, 785.

[6] Knobbe, E. T., Dunn, B., Fuqua, P. D. and Nishida, F. (1990). Appl. Opt., 29, 2729.

[7] Hermes, R. E., Allik, T. H., Chandra, S. and Hutchinson, J. A. (1993). Appl. Phys. Lett., 63, 877.

[8] Altman, J. C., Stone, P. E., Dunn, B. and Nishida, F. (1991). IEEE Photon. Tech. Lett., 3, 189.

[9] Duarte, F. J. (1994). Appl. Opt., 33, 3857.

[10] Canva, M., Georges, P., Perelgritz, J. F., Brum, A., Chaput, F. and Boilot, J. P. (1995). Appl. Opt., 34, 428.

[11] Maslyukov, A., Sokolov, S., Kaivola, M., Nyholm, K. and Popov, S. (1995). Appl. Opt., 34, 1516.

[12] Costela, A., Florido, F., García-Moreno, I., Duchowicz, R., Amat-Guerri, F., Figuera, J. M. and Sastre, R. (1995). Appl. Phys. B, 60, 383.

[13] Rodríguez, M., Costela, A., García-Moreno, I., Florido, F., Figuera, J. M. and Sastre, R. (1995). Meas. Sci. Technol., 6, 971.

[14] Duarte, F. J. (1995). Opt. Commun., 117, 480.

[15] Lam, K. S., Lo, D. and Wong, K. H. (1995). IEEE Photo. Tech. Lett., 7, 306.

[16] Costela, A., García-Moreno, I., Figuera, J. M., Amat-Guerri, F. and Sastre, R. (1996). Appl. Phys. Lett., 68, 593.

[17] Ye, C., Lam, K. S., Chik, K. P. and Lo, D. (1996). Appl. Phys. Lett., 69, 3800.

[18] Hu, W., Ye, H., Li, C., Jiang, Z. and Zhou, F. (1997). Appl. Opt., 36, 579.

[19] Chandra, S., Allik, T. A., Hutchinson, J. A., Fox, J. and Swim, C. (1997). Opt. Lett., 22, 209.

[20] Duarte, F. J., Costela, A., García-Moreno, I., Sastre, R., Ehrlich, J. J. and Taylor, T. S. (1997). Opt. Quantum Electron., 29, 461.

[21] Costela, A., García-Moreno, I., Barroso, J. and Sastre, R. (1998). J. Appl. Phys., $\mathbf{8 3}, 650$.

[22] Sastre, R. and Costela, A. (1995). Adv. Mater., 7, 198.

[23] Costela, A., García-Moreno, I., Figuera, J. M., Amat-Guerri, F. and Sastre, R. (1997). Recent Res. Devel. in Physical Chem., 1, 125. 
[24] Pacheco, D. P., Aldag, H. R., Itzkan, I. and Rostler, P. S. (1988). Proc. International Conference on Lasers' 87, Duarte, F. J. (Ed.) (STS, McLean, Va., 1988), pp. $330-337$.

[25] Delone, N. B., Interaction of Laser Radiation with Matter (Nauka, Moscow, 1989), p. 280.

[26] Dyumaev, K. M., Manenkov, A. A., Maslyukov, A. P., Matyushin, G. A., Nechitailo, V. S. and Prokhorov, A. M. (1992). J. Opt. Soc. Am. B, 9, 143.

[27] Amat-Guerri, F., Costela, A., Figuera, J. M., Florido, F. and Sastre, R. (1993). Chem. Phys. Lett., 209, 352.

[28] Ferrer, M. L., Acuña, A. U., Amat-Guerri, F., Costela, A., Figuera, J. M., Florido, F. and Sastre, R. (1994). Appl. Opt., 33, 2266.

[29] Aldoshin, M. I., Gerasimov, B. G., Manenkov, A. A. and Nechitailo, V. S. (1979). Sov. J. Quantum Electron, 9, 1102.

[30] Aldoshin, M. I., Gerasimov, B. G., Manenkov, A. A., Maslyukov, A. P., Nechitailo, V. S. and Ponomarenko, E. P. (1979). Sov. Phys.-Tech. Phys., 12, 141.

[31] Dyumaev, K. M., Manenkov, A. A., Maslyukov, A. P., Matyusin, G. A., Nechitailo, V. S. and Tsaprilov, A. S. (1982). Sov. J. Quantum. Electron., 12, 838.

[32] Barashkov, N. N. and Gunder, O. A., Fluorescent Polymers, Ser. Polymer Sci. Technol. (Ellis Horwood, Chischester, 1994), pp. 190-201.

[33] Amat-Guerri, F., Costela, A., Figuera, J. M., Florido, F., García-Moreno, I. and Sastre, R. (1995). Opt. Commun., 114, 442.

[34] Costela, A., García-Moreno, I., Figuera, J. M., Amat, F., Mallavia, R., SantaMaria, M. D. and Sastre, R. (1996). J. Appl. Phys., 80, 3167.

[35] Calderón, O. G., Guerra, J. M., Costela, A., García-Moreno, I. and Sastre, R. (1997). Appl. Phys. Lett., 70, 25.

[36] Rahn, M. D. and King, T. A. (1995). Appl. Opt. , 34, 8260.

[37] Minning, S. W., Pacheco, D. P., Burke, J. G., Dunleavy, P. V., Aldag, H. R. and Ehrlich, J. J. (1996). Proc. SPIE, 2698, 55.

[38] Kohlmannsperger, J. (1969). Z. Naturforschg., 24a, 1547.

[39] Nabolkin, Yu. V., Ogurtsova, L. A., Podgornyi, A. P., Pokrovskaya, F. S., Grigoryeva, V. I., Krasovitskii, B. M., Kutsyna, I. M. and Tishchenko, V. G. (1970). Opt. Spectrosc., 28, 528.

[40] Hänsch, T. W., Pernier, M. and Schawlow, A. L. (1971). IEEE J. Quantum Electron., QE-7, 45.

[41] Drake, J. M., Tam, E. M. and Morse, R. I. (1972). IEEE J. Quantum Electron., QE8, 92 .

[42] Ferguson, J. and Mau, A. W. H. (1972). Chem. Phys. Lett., 14, 245.

[43] Karl, N. (1972). Phys. Stat. Sol., 13, 661.

[44] Itoh, U., Takakusa, M., Moariya, T. and Saito, S. (1977). Jpn. J. Appl. Phys., 16, 1059.

[45] Zink, J. I., Dunn, B., Kaner, R. B., Knobbe, E. T. and McKiernan, J., Materials for Nonlinear Optics, Edited by Marder, S. R. Sohn, J. E. and Stucky, G. D. ACS Symposium Series, 455 (American Chemical Society, Washington, 1991), pp. 541 552.

[46] Lo, D., Parris, J. E. and Lawless, J. L. (1992). Appl. Phys. B, 55, 365.

[47] Lo, D., Parris, J. E. and Lawless, J. L. (1993). Appl. Phys. B, 56, 385.

[48] Lam, K. S., Lo, D. and Wong, K. H. (1995). Appl. Opt., 34, 3380.

[49] Lam, K. S., Lo, D. and Wong, K. H. (1995). Opt. Commun., 121, 121.

[50] Weissbeck, A., Langhoff, H. and Beck, A. (1995). Appl. Phys. B, 61, 253.

[51] Ye, C., Lam, K. S., Lam, S. K. and Lo, D. (1997). Appl. Phys. B, 65, 109.

[52] Acuña, A. U., Amat-Guerri, F., Costela, A., Douhal, A., Figuera, J. M., Florido, F. and Sastre, R. (1991). Chem. Phys. Lett., 187, 98.

[53] Costela, A., García-Moreno, I., Figuera, J. M., Amat-Guerri, F., Barroso, J. and Sastre, R. (1996). Opt. Commun., 130, 44.

[54] Bos, F. (1981). Appl. Opt., 20, 3553. 